\title{
Una propuesta para mejorar la experiencia de los adultos mayores con las redes sociales
}

\author{
Claudia Cardozo \\ claudia_yoryi@yahoo.com.ar \\ Adriana Martín \\ adrianaelba.martin@gmail.com \\ Viviana Saldaño \\ vsaldanio@gmail.com \\ Gabriela Gaetán \\ gabrielagaetan@yahoo.com.ar \\ Integrantes del Grupo de Investigación y Formación en Ingeniería de \\ Software (GIFIS) del Instituto de Tecnología Aplicada (ITA) de la Universidad \\ Nacional de la Patagonia Austral (UNPA)-Unidad Académica Caleta Olivia (UACO)
}

\section{Extracto}

Las redes sociales son una de las aplicaciones más solicitadas desde los dispositivos móviles. En este escenario, el diseño de interfaces de usuario (IU) se enfrenta a un nuevo desafío y, muy particularmente, al considerar a la población de adultos mayores (AM). En este trabajo, se presentan un conjunto de 23 recomendaciones de diseño para mejorar la experiencia de los usuarios AM con las redes sociales usando tablets. Posteriormente, se define y aplica un ciclo de prototipado, el cual está basado en una customización de la metodología Lean UX (user experience [experiencia de usuario]) y dirigido por nuestras recomendaciones de diseño. El proceso de desarrollo, que está centrado en las necesidades y preferencias de la población de AM, propone un diseño alternativo de red social para tablet. Así, el prototipo resultante está enfocado en mejorar la experiencia de interacción y, por ende, también en propiciar la integración social virtual de este grupo creciente de ciudadanos.

Palabras clave: adultos mayores (AM); redes sociales; experiencia de usuario (user experience [UX]); usabilidad; recomendaciones de diseño; tablet. 


\title{
A proposal for improving the experience of older adults with social networks
}

Claudia Cardozo

Adriana Martín

Viviana Saldaño

Gabriela Gaetán

\begin{abstract}
Social networks are one of the most requested applications from mobile devices. In this scenario, interface design (IU) faces a new challenge, and very particularly, when considering the older adult population (OA). In this work, we present a set of 23 design recommendations to improve the experience of OA in social networks using tablet. Then, a prototyping cycle is defined and applied, which is based on a customization of Lean UX (user experience) methodology and driven by our design recommendations. The development process, which is centered on the needs and preferences of the OA population, proposes an alternative social network design for tablet. Thus, the resulting prototype is focused on improving the interaction experience, and therefore, also promoting the virtual social integration of this growing group of citizens.
\end{abstract}

Keywords: older adults (OA); social networks; user experience (UX); usability; design recommendations; tablet.

Citation: Cardozo, C., Martín, A., Saldaño, V. y Gaetán, G. (2020). A proposal for improving the experience of older adults with social networks. Tecnología, Ciencia y Educación, 16, 113-142. 


\section{Sumario}

1. Introducción

2. Antecedentes

2.1. Accesibilidad web-usabilidad-UX

2.2. Herramientas de prototipado

2.3. Metodologías ágiles y UX: Lean UX

3. Nuestra propuesta

3.1. Recomendaciones de diseño

3.2. CP centrado en AM

3.3. Aplicación del CP centrado en AM

3.3.1. Definición de la muestra

3.3.2. Ejecución paso a paso del CP centrado en AM

4. Resultados obtenidos y discusión

5. Conclusiones y trabajos futuros

Referencias bibliográficas 


\section{Introducción}

El uso de los dispositivos móviles ha experimentado un auge extraordinario y plantea un nuevo escenario desde el cual las personas pueden acceder a la web con solo disponer de una conexión a internet. Las redes sociales son una de las aplicaciones más solicitadas desde dispositivos móviles, $y$, en particular, la creciente población de ciudadanos AM manifiesta interés en el uso de tablets.

\section{Las redes sociales son una de las aplicaciones más solicitadas desde dispositivos móviles, $\mathrm{y}$, en particular, la creciente población de ciudadanos AM manifiesta interés en el uso de tablets} En este contexto, el diseño de IU se enfrenta a un nuevo desafío que requiere de esfuerzos conjuntos para abarcar todo el espectro de factores que pueden influir en el producto resultante, tales como las características propias del perfil de usuarios destinatarios; del producto web que se va a considerar; del dispositivo preferentemente seleccionado para el despliegue; de los aspectos demográficos, poblacionales, geográficos y regionales; etc. Estos factores ponen en evidencia la complejidad inherente al diseño de interfaces que consideren la UX. En la práctica, los desarrolladores de IU deben tomar decisiones de diseño para satisfacer conjuntamente más de uno de estos factores señalados.

Nuestro grupo de investigación ha estado trabajando fuertemente con usuarios AM (Cardozo, Martín y Saldaño, 2017a, 2017b; Saldaño, Gaetán, Martín y Cardozo, 2018a, 2018b; Saldaño, Martín, Gaetán y Vilte, 2013; Saldaño, Martín, Gaetán y Cardozo, 2016; Vilte, Saldaño, Gaetán y Martín, 2015). Específicamente, nos hemos enfocado en el uso de las redes sociales por parte de este grupo creciente de usuarios, ya que este tipo de aplicaciones constituyen un instrumento digital vital para la integración ciudadana, social y también familiar, asistiendo a la superación de brechas generacionales y geográficas. Si bien el uso de las redes sociales se relaciona con una costumbre propia de la gente joven, existen estudios (Barroso y Aguilar, 2017; Duggan, 2015; Luna, Mendoza y Álvarez, 2015) que vienen revelando que los AM constituyen un nicho importante de mercado, aunque, normalmente, no del todo considerado por las aplicaciones de redes sociales. Otros trabajos y sondeos estadísticos provenientes de centros de investigación (Leme, Zaina y Casadei, 2015; Lu, 2017) también indican el aumento visible del número de personas mayores de 60 años que interactúan desde dispositivos móviles. Posicionados desde esta realidad, hemos identificado que nuestros $A M$ no son ajenos a esta tendencia mundial $y$, en particular, cuando se trata de acceder a una red social desde un dispositivo móvil, prefieren hacerlo desde una tablet. 
Haciendo una revisión exhaustiva del estado de la cuestión, se han realizado esfuerzos, ya sea por parte de grupos de investigación (Cornejo, Weibel, Tentori y Favela, 2015; Marcelino, Laza y Pereira, 2016; Miranda, Martín, Saldaño y Gaetán, 2014; Sulmont, Pontual y Moffatt, 2018) como por parte de organismos gubernamentales y privados (Peñalver, 2016; Presidencia de la Nación, 2016), por reducir la brecha digital para la integración social de los AM. Por ejemplo, en Sulmont et al. (2018) se diseña una interfaz de usuario alternativa para Facebook en la que se implementan los siguientes cambios de diseño: filtrar contenido en noticias, minimizar componentes de diseño, facilitar la navegación, aumentar la accesibilidad y reconocer configuraciones y páginas de privacidad. Se realizó una evaluación con tres AM que evidenciaron mejoras alcanzadas con el diseño de interfaz y confirmaron las barreras de alto nivel para el uso de Facebook, especialmente relacionadas con la capacidad de aprendizaje.

En Miranda et al. (2014), los esfuerzos están enfocados en analizar barreras de accesibilidad y usabilidad a las que se enfrentan los AM cuando interactúan con Facebook. Los autores diseñan un experimento completo basado en la técnica card sorting, logrando obtener una IU que surge de la participación activa de los AM. Los AM definen una distribución y apariencia a la medida de sus necesidades. Dado que la propuesta ofrece un boceto, es necesario plasmar los hallazgos utilizando alguna herramienta para que los AM experimenten con el diseño de IU propuesto desde algún tipo de dispositivo.

Por otra parte, como se puede ver en Peñalver (2016), la Fundación Tecnologías Sociales (TECSOS) ${ }^{1}$, Cruz Roja Española ${ }^{2}$ y la Fundación Vodafone España ${ }^{3}$ presentan el proyecto Enred@te. Esta propuesta permite acceder a una red conformada por AM y voluntarios de la Cruz Roja con el objetivo básico de fortalecer las relaciones habituales en el contexto de la atención y el apoyo. La aplicación es accesible a través de tablets con sistema operativo Android (para los $\mathrm{AM}$ ) y de ordenadores personales (para los voluntarios). Debido a que esta es una iniciativa propia de Cruz Roja Española, el acceso está circunscripto solo a los AM pertenecientes a dicha organización.

\footnotetext{
$1<$ https://fundaciontecsos.es/es>.

$2<$ https://www.cruzroja.es/principal/web/cruz-roja/inicio>.

$3<$ http://www.fundacionvodafone.es/>.
} 
En Gomes, Duarte, Coelho y Matos (2014) se identifican recomendaciones para diseñar redes sociales dirigidas a AM y son aplicadas en la creación de prototipos de interfaz para acceder a los servicios de Facebook desde tablets. De esta manera se logra mayor aceptación en comparación con la aplicación nativa de Facebook. Sin embargo, se detecta que es necesario realizar un análisis más exhaustivo para identificar un mayor número de recomendaciones que puedan ser aplicadas para elaborar los prototipos de interfaz correspondientes a las tareas más usadas por los AM. Se considera que el diseño de los prototipos de interfaz debe ser iterativo e incremental, permitiendo que los usuarios AM participen activamente en distintas instancias del mismo hasta lograr la producción final del prototipo de interfaz. Dado que esta es una propuesta alternativa y diferente a la aplicación nativa de Facebook, se destaca que la apariencia presentada difiere en aspectos como menú de selección, vocabulario, iconografía, distribución de elementos y colores usados, lo que podría ser una dificultad en el aprendizaje del uso de la red social por parte del AM y su entorno familiar.

Finalmente, en Presidencia de la Nación (2016) se presenta +Simple, una plataforma diseñada especialmente para los AM, la cual permite un fácil acceso a diferentes servicios ya existentes, tales como cultura, trámites, redes sociales, salud, etc. Esta es una iniciativa nacional y el acceso a este beneficio se concreta de acuerdo al caso particular de cada AM solicitante. Dado que la plataforma +Simple actúa solo como un portal de acceso a aplicaciones de servicios existentes, a partir de que el AM ingresa a estos servicios, debe interactuar con las IU de origen que ofrecen estas aplicaciones.

Basándonos en los antecedentes, en los trabajos relacionados y en la experiencia recabada durante años de investigación y producción en el área de diseño de IU, en este trabajo se propone, en primera instancia, un conjunto de 23 recomendaciones de diseño destinadas a mejorar la experiencia de los AM con Facebook y desde tablets. Posteriormente, en segunda instancia, se define y aplica un ciclo de prototipado dirigido por nuestras recomendaciones de diseño y basado en los principios de la metodología ágil Lean UX (Gothelf y Seiden, 2014).

El ciclo iterativo-incremental propuesto, el cual utiliza dos herramientas de prototipado (de baja y de alta fidelidad), prioriza un desarrollo centrado en las necesidades y preferencias de los AM. El prototipo resultante propone un diseño alternativo de red social para tablet, mejorando la experiencia de los usuarios AM y, por ende, propiciando la integración social virtual de este grupo creciente de ciudadanos.

\section{Antecedentes}

Las características que diferencian los dispositivos móviles de los ordenadores convencionales, tales como el tamaño, el tipo de interacción, la ubicuidad, entre otras, requieren que 
el diseño de IU proporcione una buena UX que considere dichas características. Tal como se describe en Flamarich (2013), existen valiosos recursos que contribuyen al diseño de IU para dispositivos móviles. A continuación, se presentan brevemente solo algunos de los antecedentes que se han estudiado y analizado en profundidad durante esta investigación para dar soporte a este trabajo de investigación.
Las características que diferencian los dispositivos móviles de los ordenadores convencionales requieren que el diseño de IU proporcione una buena UX que considere dichas características

\subsection{Accesibilidad web-usabilidad-UX}

Según Nielsen Norman Group 4 , la «UX» es un concepto integrador de todos los aspectos de la interacción entre el usuario final y la compañía, sus servicios y productos. Si bien esta es solo una de las numerosas definiciones que existen acerca de un concepto no trivial, como lo es la UX, su lectura pone en evidencia que para abordar el tratamiento de la UX es necesario un enfoque fundamentalmente interdisciplinario.

La usabilidad y accesibilidad web son propiedades de calidad que contribuyen fuertemente a mejorar la UX. La accesibilidad web, cuyo referente máximo es el World Wide Web Consortium $(\mathrm{W} 3 \mathrm{C})^{5}$, se enfoca en facilitar a las personas el acceso e interacción al contenido web.

Las recomendaciones de accesibilidad $(W 3 C, 2018)$ proporcionan técnicas de uso del markup language para desarrollar IU con código bien formado y accesible. Por otra parte, la usabilidad, cuyo referente máximo es Jakob Nielsen, se enfoca en alcanzar la facilidad de uso de las IU por parte de los usuarios web. Las recomendaciones de usabilidad asisten al diseño IU considerando diferentes aspectos (Budiu y Nielsen, 2015; Nielsen, 2013; Pernice, Estes y Nielsen, 2013).

Sin lugar a dudas, la usabilidad y accesibilidad web constituyen herramientas conceptuales que hay que considerar cuando el objetivo es crear una UX satisfactoria, limitada a las dimensiones de un dispositivo móvil.

\section{Sin lugar a dudas, la usabilidad y accesibilidad web constituyen herramientas conceptuales que hay que considerar cuando el objetivo es crear una UX satisfactoria, limitada a las dimensiones de un dispositivo móvil}

\footnotetext{
$4<$ https://www.nngroup.com/people/jakob-nielsen/>.

$5<$ https://www.w3.org>.
} 


\subsection{Herramientas de prototipado}

El prototipo es una excelente herramienta que permite comunicar la intención de un diseño de forma práctica, clara y efectiva. Entre sus usos más comunes se incluyen trabajar en el diseño, evaluar la usabilidad e, incluso, evaluar la factibilidad técnica y económica de un producto (Warfel, 2009). Es importante determinar cuál es la audiencia y el objetivo del prototipo para definir con qué fidelidad es necesario prototipar. De acuerdo a la fidelidad del diseño de IU resultante, los prototipos pueden ser de «baja fidelidad» o de «alta fidelidad»; pero también, de acuerdo a la capacidad de ejecución del diseño de IU resultante, los prototipos pueden ser «ejecutables» o «no ejecutables». Las herramientas de prototipado se han vuelto muy populares en el campo de la UX, ya que permiten una interacción en tiempo real con los usuarios, poniéndolos en el centro del diseño.

Algunos ejemplos de herramientas de prototipado son Balsamiq Mockup ${ }^{6}$ (wireframing estáticos, con licencia, que proporciona prototipos de baja fidelidad), Pencil Project ${ }^{7}$ (soporte wireframing open source que proporciona prototipos de alta fidelidad) y Axure $\mathrm{RP}^{8}$ (wireframing interactivos, con licencia, que proporciona prototipos de alta fidelidad/ejecutables).

\subsection{Metodologías ágiles y UX: Lean UX}

A lo largo de los años, las metodologías de desarrollo utilizadas en el entorno digital han evolucionado sustancialmente para dar soluciones a los cambios del sector (Amaya, 2013). Algunos de los disparadores que propiciaron el diseño y desarrollo ágil son los productos más orientados a la web, la proliferación de los dispositivos móviles, el crecimiento notorio del movimiento open source y, más recientemente, el creciente interés en considerar la UX de los usuarios.

Particularmente, las propuestas basadas en ciclos ágiles resultan muy adecuadas para diseñar y desarrollar pensando en las necesidades y en las preferencias de los usuarios.

Lean UX (Gothelf y Seiden, 2014) ofrece una perspectiva completa de cómo los principios de Lean Startup (Ries, 2011) pueden aplicarse al contexto de la UX, conjugándolos con los conceptos de customer development (Blank, 2013), design thinking (Brown, 2008) y las

\footnotetext{
$6<$ https://balsamiq.com>.

$7<$ https://pencil.evolus.vn>.

$8<\mathrm{https} / / /$ www.axure.com>.
} 
metodologías de desarrollo ágil de software. La figura 1 ilustra el proceso propuesto por Lean UX. En el primer paso, se declara un conjunto de «suposiciones» (assumptions), las cuales le permiten al equipo del proyecto crear un punto de partida común. Esta declaración de supuestos saca las ideas de todos, revelando la divergencia de opiniones y exponiendo un amplio conjunto de posibles soluciones. Lean UX propone priorizar estas suposiciones a través de plantear «hipótesis» (hypotheses). La priorización entrega un conjunto de "declaraciones» (statements) que capturan los aspectos que hay que considerar por el diseño y que son lo suficientemente específicas como para ser probadas, y constituyen la hoja de ruta para disparar un ciclo de diseño colaborativo, ágil e iterativo-incremental (Gothelf y Seiden, 2014).

Figura 1. El proceso Lean UX

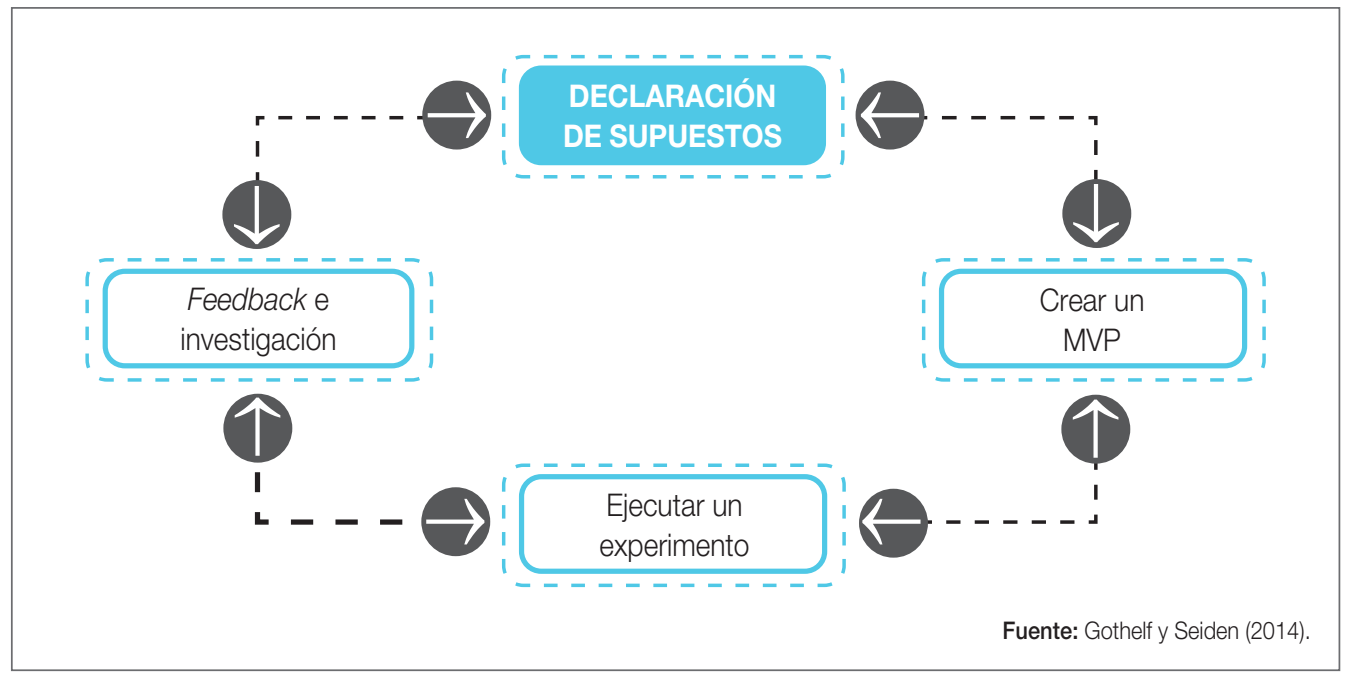

\section{Nuestra propuesta}

Los dispositivos de tipo tablet constituyen la alternativa más adecuada para los AM, principalmente, debido a las dimensiones de la pantalla táctil. Sin embargo, al interactuar con las redes sociales desde estos dispositivos, los AM ven muchas veces frustradas sus expectativas de uso debido a que el diseño de IU y la funcionalidad ofrecidos no responden a sus necesidades.
Los dispositivos de tipo tablet constituyen la alternativa más adecuada para los $\mathrm{AM}$ debido a las dimensiones de la pantalla táctil. $\mathrm{Al}$ interactuar con las redes sociales desde estos dispositivos, los AM ven muchas veces frustradas sus expectativas de uso porque el diseño de IU y la funcionalidad ofrecidos no responden a sus necesidades 


\subsection{Recomendaciones de diseño}

Enfocados en mejorar la UX de los usuarios $\mathrm{AM}$ con las redes sociales y desde tablets, y como resultado de un proceso de análisis y sistematización llevado a cabo durante sucesivos trabajos de investigación e interacción con los usuarios AM (Cardozo et al., 2017a, 2017b), finalmente, se propone un conjunto de 23 recomendaciones de diseño (RD). La figura 2 ilustra el proceso que se ha estado aplicando en la identificación y desarrollo de este conjunto de RD.

Figura 2. Proceso de análisis y sistematización

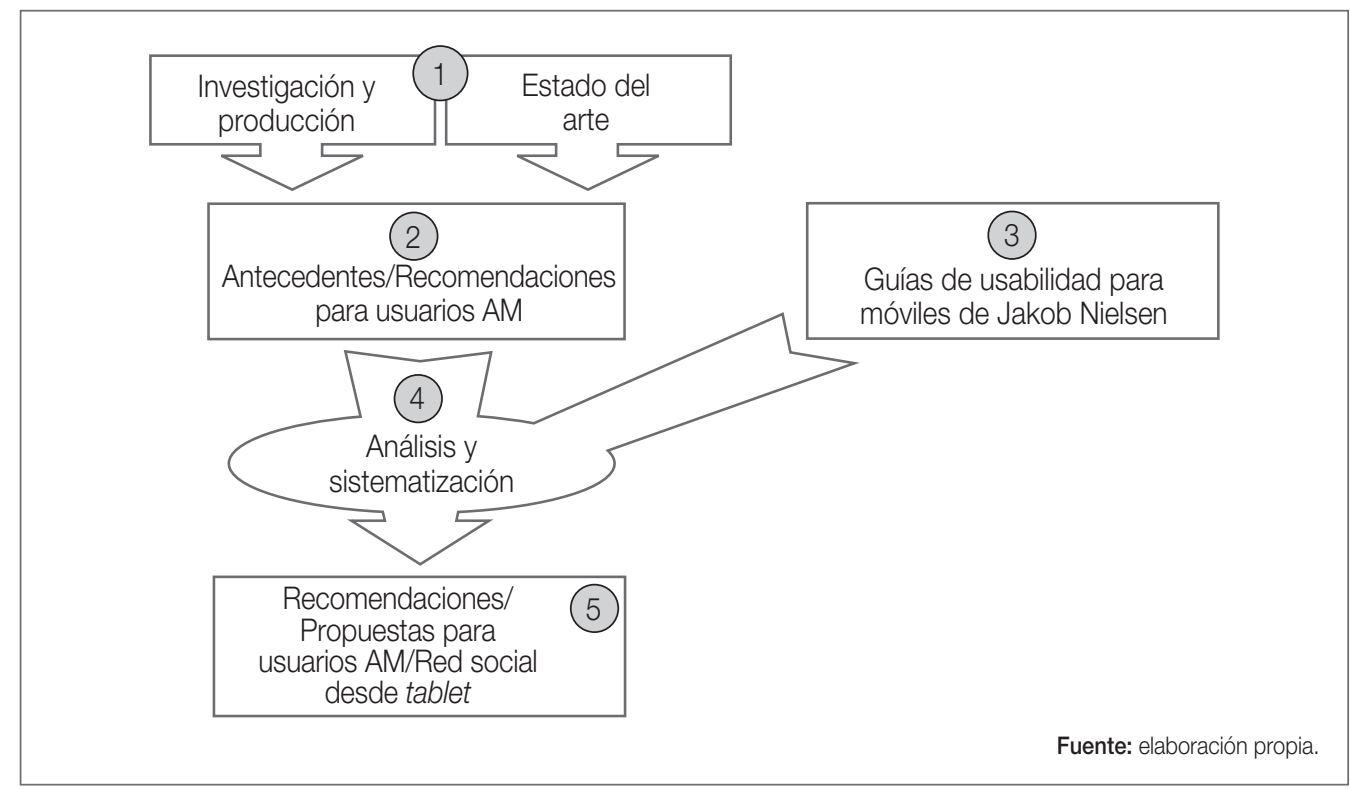

En la figura 2 (paso 1), la experiencia recabada tras años de investigación enfocada en el diseño de IU usables y accesibles, y, en particular, dirigida a la población AM, conjuntamente con una revisión exhaustiva del estado de la cuestión, constituyen la entrada (paso 2) al proceso; mientras que en el paso 3, las guías de diseño de reconocidos referentes en áreas de usabilidad (Budiu y Nielsen, 2015; Nielsen, 2013; Pernice et al., 2013) proveen una entrada adicional para mejorar la UX. De esta manera, las entradas (pasos 2 y 3 ) proveen contexto y soporte al proceso. Sobre esta sólida base de conocimientos de entrada, en el 
paso 4 se realiza un proceso de análisis y sistematización dirigido por la experiencia recopilada durante años de trabajo con usuarios AM. Finalmente, en el paso 5, el resultado es un conjunto de $23 \mathrm{RD}$ específicamente dirigidas a usuarios AM para mejorar la experiencia en el acceso e interacción con las redes sociales y desde tablets. El conjunto resultante de las 23 RD para usuarios AM, aplicables a IU de redes sociales y desde tablets, se organiza dentro de las siguientes cinco categorías:

1. Contenido.

2. Organización de la página.

3. Presentación de la información.

4. Vínculos y navegación del sitio.

5. Áreas y elementos objetivo.

Debido a cuestiones de espacio, en el cuadro 1 únicamente se presentan las RD correspondientes a la categoría 5.

\section{Cuadro 1. Categoría 5 (áreas y elementos objetivo)}

\section{Dificultad experimentada por los AM}

Dificultad para recordar la funcionalidad asociada a los distintos iconos utilizados en las interfaces de las aplicaciones web.

Disminución de la capacidad motora, que dificulta la selección de áreas objetivo.

Disminución de la capacidad visual y dificultad para reconocer las áreas objetivo

Disminución de capacidades visual y motora.

\section{Recomendación}

5.1. Los iconos utilizados en la interfaz deben ser iconos estándar, utilizados en otras aplicaciones. En caso de crearse iconos nuevos, estos deben asemejarse a elementos conocidos de la vida cotidiana (diseño skeumorphism).

5.2. Limitar la cantidad de áreas objetivo a 10 por pantalla de la interfaz.

5.3. En los dispositivos táctiles, los elementos que puedan ser objetivo deben tener un diseño acorde que sugiera la tarea que hay que ejecutar; por ejemplo, iconos/botones con apariencia 3D (diseño affordance).

5.4. Las áreas objetivo deben ocupar una superficie mínima de $1,5 \mathrm{~cm}$ (ancho) $x 1 \mathrm{~cm}$ (alto) y tener una distancia de separación mínima de $1 \mathrm{~cm}$ entre ellas.

5.5. Para las tareas más usadas, complementar los diseños skeumorphism y affordance con un texto descriptivo que permita reforzar la correcta comprensión de los mismos.
Disminución de la capacidad visual, dificultad para recordar la funcionalidad asociada a distintos iconos y dificultad para reconocer áreas objetivo. 


\subsection{CP centrado en $A M$}

Con el propósito de aplicar nuestras RD, se define un ciclo de desarrollo que propone diseños tempranos e incrementales con la participación activa de los usuarios AM. La figura 3 ilustra el ciclo de prototipado (CP) propuesto, que está centrado en los usuarios $\mathrm{AM}$, basado en la metodología ágil Lean UX y conducido por nuestras RD, las cuales guían la ejecución de todos los pasos (y sus respectivas actividades).

Con el propósito de aplicar nuestras $\mathrm{RD}$, se define un ciclo de desarrollo que propone diseños tempranos e incrementales con la participación activa de los usuarios $\mathrm{AM}$

Figura 3. CP centrado en AM

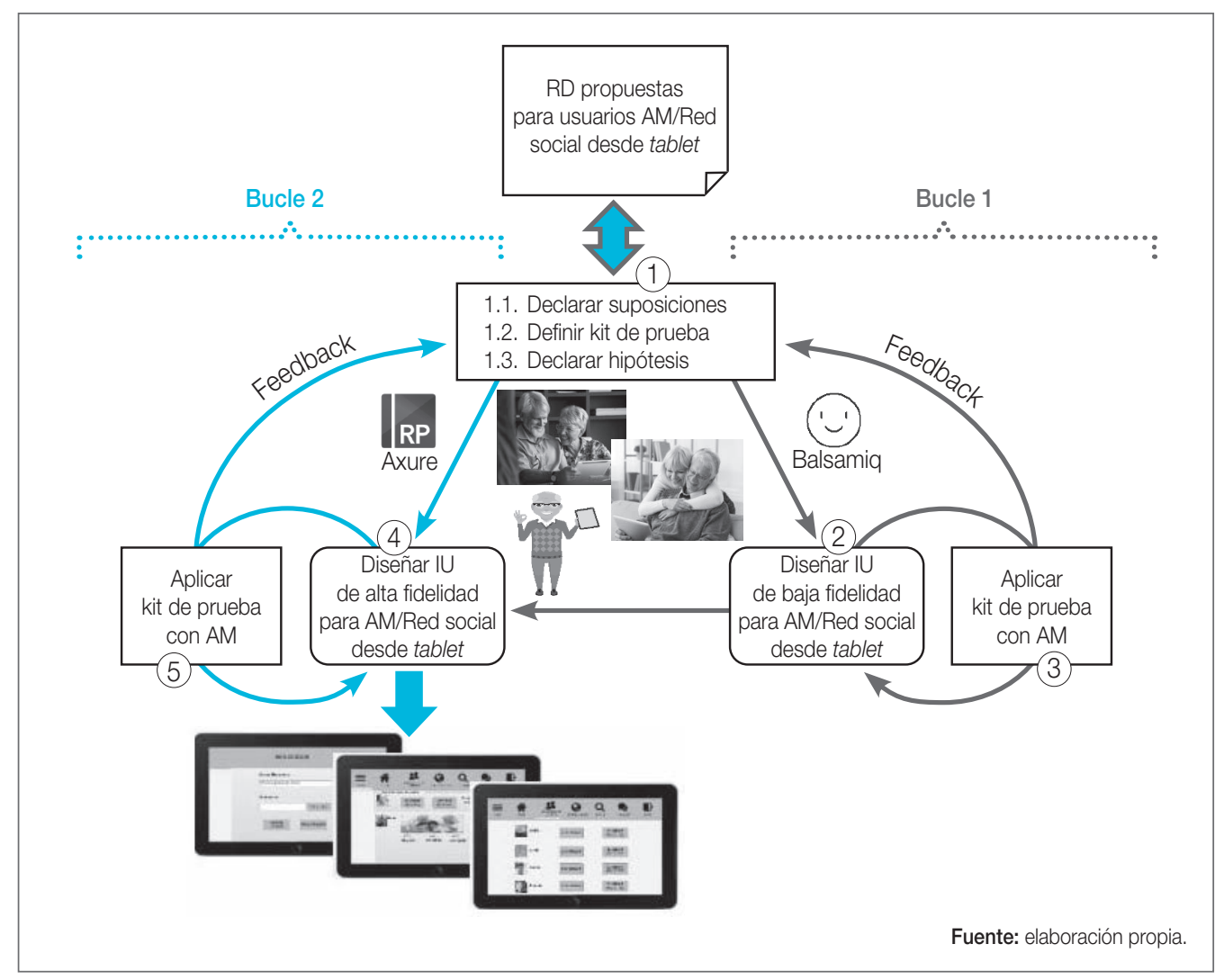

Tal como ilustra la figura 3, las RD son la única «entrada» al CP. A continuación, describimos brevemente cada uno de los pasos que componen el CP: 
- Paso 1. Este paso es crucial en la ejecución del CP, ya que es central de los siguientes dos bucles iterativos e incrementales que permiten desarrollar:

- Bucle 1 (pasos 1, 2 y 3): un prototipo de baja fidelidad.

- Bucle 2 (pasos 1, 4 y 5): un prototipo de alta fidelidad.

En nuestro CP, el paso 1 de la metodología Lean UX (Gothelf y Seiden, 2014) se customiza en tres actividades:

- 1.1. Declarar suposiciones.

- 1.2. Definir kit de prueba.

- 1.3. Declarar hipótesis.

En la actividad 1.1, se declara una lista de suposiciones acerca de las características y funcionalidades ofrecidas en la IU de la red social Facebook. Debido a que la declaración de la lista de suposiciones está guiada por nuestras RD (desarrolladas sobre las necesidades y preferencias de los usuarios AM), todas las suposiciones deben ser consideradas en el desarrollo de los prototipos y, por lo tanto, se omite la instrumentación de la matriz de priorización de suposiciones propuesta por la metodología Lean UX. A partir de cada suposición, en la actividad 1.3, se declara una lista de hipótesis; y debido a que la declaración de cada hipótesis a partir de cada suposición debe incluir un mecanismo de validación, en la actividad 1.2 de nuestro CP se define un kit de prueba. Este kit de prueba se compone de un conjunto de tareas, las cuales permitirán disparar la interacción entre los usuarios AM y los diseños de IU propuestos por los prototipos de baja y de alta fidelidad. Es importante señalar que los usuarios AM en los que se centra el CP se modelan aplicando el método de protopersonas, tal como propone Lean UX (Gothelf y Seiden, 2014).

Lean UX utiliza funciones para satisfacer las necesidades de los usuarios. En nuestra propuesta, las tareas son las encargadas de alcanzar este objetivo. A continuación, de describen los bucles 1 y 2, ambos con origen en el paso 1 de nuestro CP.

- Bucle 1 (pasos 1, 2 y 3 del CP). En el paso 2, se propone un primer diseño de IU con un prototipo de baja fidelidad usando la herramienta Balsamiq Mockups. A continuación, en el paso 3, el prototipo de baja fidelidad es evaluado por los usuarios AM, quienes interactúan ejecutando el conjunto de tareas que componen el kit de prueba. El feedback obtenido permite validar la lista de hipótesis y determinar la continuidad (o no) del prototipo propuesto. De acuerdo al resultado obtenido en la validación de las hipótesis, se procede a mejorar el prototipo o se plantea la revisión de la lista de suposiciones, según corresponda. El entregable que resulta de ejecutar el bucle 1 es un prototipo de baja fidelidad final. 
- Bucle 2 (pasos 1, 4 y 5 del CP). En el paso 4, con base en el prototipo de baja fidelidad final, se propone un segundo diseño de IU con un prototipo de alta fidelidad, usando la herramienta Axure RP. A continuación, en el paso 5, el prototipo de alta fidelidad es evaluado por los usuarios $A M$, quienes interactúan ejecutando el conjunto de tareas que componen el kit de prueba. Nuevamente, el feedback obtenido determina la permanencia en el bucle y las acciones que hay que tomar durante la continuidad de la ejecución del mismo. El entregable que resulta de ejecutar el bucle 2 es un prototipo de alta fidelidad final y constituye la «salida» del CP. Es importante destacar que los pasos 2, 3 y 4 de la metodología Lean UX (Gothelf y Seiden, 2014) son customizados e implementados por los bucles 1 y 2 del CP.

\subsection{Aplicación del CP centrado en AM}

Para comenzar con la aplicación del CP centrado en AM, resulta pertinente realizar una breve, pero no por ello menor, caracterización del grupo de usuarios: los AM. A tal efecto, a continuación, explicamos el origen de la muestra.

\subsubsection{Definición de la muestra}

Los usuarios AM que participaron en este proyecto del GIFIS (UNPA, 2010) son alumnos que asistieron a los talleres de computación desarrollados en el marco del programa Universidad para Adultos Mayores Integrados (UPAMI) (Instituto Nacional de Servicios Sociales para Jubilados y Pensionados, 2017) en la UNPA (ciudad de Caleta Olivia). El rango de edad oscila entre 65 y 85 años.

Se definió realizar diversas réplicas del taller (Saldaño et al., 2018a) debido a que la cantidad de AM en la ciudad de Caleta Olivia es escasa (Instituto Nacional de Estadística y Censos de la República Argentina [INDEC], 2019), y más aún los AM interesados en participar en la propuesta. Cabe destacar que los diversos talleres dictados por el GIFIS para este sector de la población, en promedio, convocan inicialmente a 10 AM por cada taller, disminuyendo la cantidad de AM que logran finalizar. Debido a esto, de los 10 AM que inician cada taller, en este proyecto solo participaron en las pruebas aquellos que lograron culminar cada taller. De esta manera, a través de las diversas réplicas del taller (Saldaño et al. , 2018a) se pudo reunir una cantidad de AM para obtener una muestra representativa.

A los efectos de determinar la cantidad de los AM que debían participar en la realización del kit de prueba, y utilizando los datos provistos por el Censo Nacional de la Población de 2010 de Argentina (INDEC, 2019), se trabajó sobre las siguientes consideraciones:

- El total de población con 65 años o más en la ciudad de Caleta Olivia es de 2.718 personas. 
- En la provincia de Santa Cruz, se analizó el uso de ordenadores por parte de la población con 65 años o más, como se observa en el cuadro 2 (donde el rango de edad para esta franja etaria se inicia en los 60 años).

- Particularmente, para la ciudad de Caleta Olivia, los datos provistos corresponden al departamento Deseado, el cual incluye las ciudades de Puerto Deseado, Caleta Olivia, Cañadón Seco, Jaramillo, Koluel Kaike, Las Heras y Pico Truncado. A tal efecto, se analizan los datos provistos por el Censo Nacional de la Población de 2010 de Argentina (INDEC, 2019):

- Población AM del departamento Deseado: 5.055 personas.

- Población AM que usa ordenadores en el departamento Deseado: 1.239 personas (de acuerdo al cuadro 2).

Cuadro 2. Uso de ordenador personal según edad

\begin{tabular}{|c|c|c|c|}
\hline \multicolumn{4}{|c|}{$\begin{array}{c}\text { Cuadro P8-D. Provincia de Santa Cruz/Departamento Deseado/ } \\
\text { Población de } 3 \text { años y más en viviendas particulares por utilización de } \\
\text { computadora según edad (año 2010) }\end{array}$} \\
\hline \multirow{2}{*}{ Edad } & \multirow{2}{*}{$\begin{array}{c}\text { Población de } 3 \text { años y más } \\
\text { en viviendas particulares }\end{array}$} & \multicolumn{2}{|c|}{ Utilización de computadora } \\
\hline & & Sí & No \\
\hline Total & 98.925 & 65.355 & 33.570 \\
\hline $3-5$ & 6.665 & 3.189 & 3.476 \\
\hline 6 & 2.184 & 1.506 & 678 \\
\hline 7 & 2.103 & 1.571 & 532 \\
\hline 8 & 2.016 & 1.631 & 385 \\
\hline 9 & 2.012 & 1.677 & 335 \\
\hline 10 & 2.151 & 1.870 & 281 \\
\hline 11 & 2.016 & 1.814 & 202 \\
\hline 12 & 2.067 & 1.844 & 223 \\
\hline 13 & 1.952 & 1.751 & 201 \\
\hline 14 & 2.039 & 1.802 & 237 \\
\hline $15-19$ & 9.747 & 8.463 & 1.284 \\
\hline $20-24$ & 9.099 & 6.906 & 2.193 \\
\hline $25-29$ & 9.688 & 6.995 & 2.693 \\
\hline $30-34$ & 9.735 & 6.947 & 2.788 \\
\hline $35-39$ & 7.893 & 5.348 & 2.545 \\
\hline
\end{tabular}




\begin{tabular}{|c|c|c|c|c|}
\hline \multicolumn{5}{|c|}{$\begin{array}{l}\text { Cuadro P8-D. Provincia de Santa Cruz/Departamento Deseado/ } \\
\text { Población de } 3 \text { años y más en viviendas particulares por utilización de } \\
\text { computadora según edad (año 2010) }\end{array}$} \\
\hline \multirow{2}{*}{ Edad } & \multirow{2}{*}{$\begin{array}{c}\text { Población de } 3 \text { años y más } \\
\text { en viviendas particulares }\end{array}$} & \multicolumn{3}{|c|}{ Utilización de computadora } \\
\hline & & \multicolumn{2}{|l|}{ Sí } & No \\
\hline \multicolumn{5}{|l|}{ 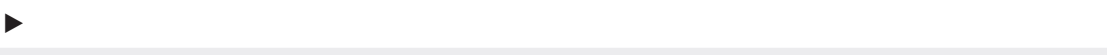 } \\
\hline $40-49$ & 11.842 & \multicolumn{2}{|l|}{7.214} & 4.628 \\
\hline $50-59$ & 8.219 & \multicolumn{2}{|l|}{3.588} & 4.631 \\
\hline $60-69$ & 4.403 & 976 & & 3.427 \\
\hline $70-79$ & 2.340 & 226 & 1.239 & 2.114 \\
\hline 80 y más & 754 & 37 & & 717 \\
\hline
\end{tabular}

Fuente: INDEC (2019).

Teniendo presente que la población AM en la ciudad de Caleta Olivia es de 2.718 personas, lo que representa aproximadamente el $54 \%$ de la población AM del departamento Deseado, es posible realizar una estimación porcentual de la población AM que en Caleta Olivia utiliza ordenadores, siendo este valor de 669 personas.

- A partir de los 669 AM que usan ordenadores es posible identificar, según registros internos del GIFIS, que la cantidad de AM que se interesaron y asistieron a los talleres del UPAMI en el periodo 2016-2019 (se considera este periodo porque a partir del año 2016 se comenzó a trabajar con los AM teniendo presente el objetivo de esta tesis) fue de 60 personas.

A continuación, en la figura 4 se presentan gráficamente los datos explicados anteriormente.

Figura 4. Población de AM que asistió a los talleres del UPAMI en Caleta Olivia (2016-2019)

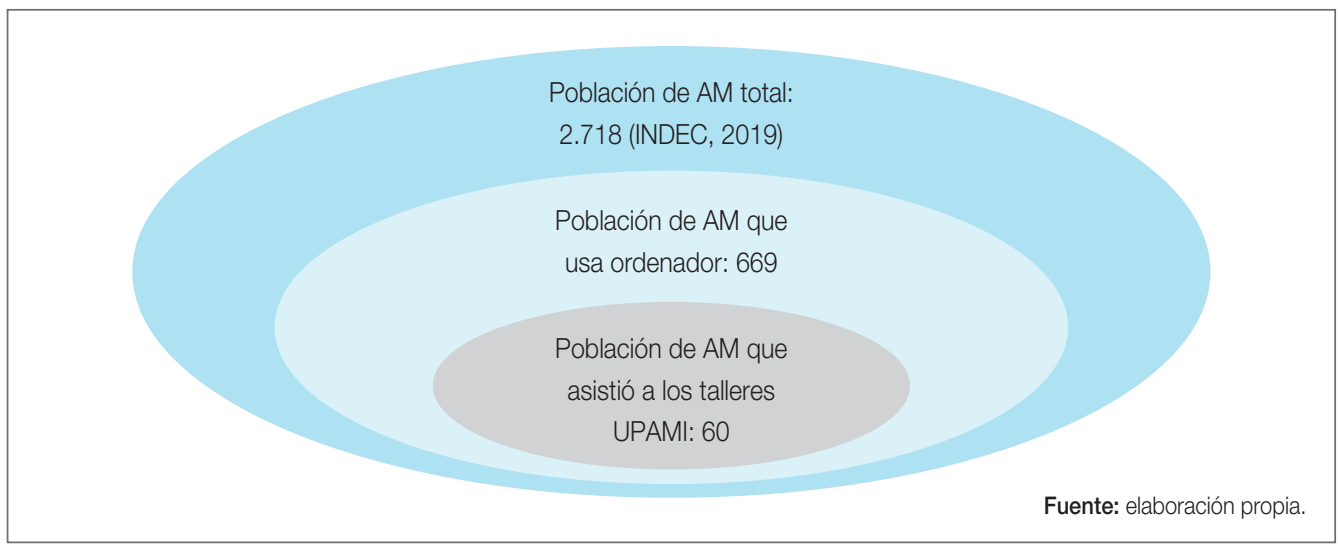


A partir de estos datos se determina el tamaño que debe tener la muestra de AM para que la misma sea representativa de la población en estudio. Para ello se aplica la siguiente fórmula estadística (véase ecuación 1), que permite determinar el tamaño de una muestra de población finita (Vivanco, 2005).

Ecuación 1. Tamaño de la muestra de población finita

$$
n=\frac{Z^{2} \times N \times p \times q}{e^{2} \times(N-1)+Z^{2} \times p \times q}
$$

Donde:

- $\quad Z$ = Coeficiente de confiabilidad.

- $\quad N=$ Tamaño de la población o universo.

- $p=$ Proporción de éxito.

- $q=$ Proporción de fracaso $(q=1-p)$.

- $e=$ Margen de error.

Al aplicar la fórmula se consideraron los siguientes datos:

- $\quad$ Nivel de confianza $=90 \%$ y $Z=1,645$.

- $N=60$.

- $p=0,50$.

- $q=0,50$.

- $e=10 \%$.

A partir de los cálculos realizados se obtiene una muestra representativa de la población AM interesada en el uso de ordenadores, con un nivel de confianza del $90 \%$ y un margen de error del $10 \%$. Se concluye que se requiere realizar el kit de prueba a 32 AM. Por lo tanto, fueron convocados AM que participaron en los talleres desarrollados por el GIFIS y que usaban y/o manifestaban interés por las redes sociales. Al ejecutar el CP, nuestro propósito es desarrollar un diseño alternativo de red social para Facebook, enfocado en mejorar la UX de los AM desde tablets. Por lo tanto, en primera instancia, se analiza una red social ya existente (Facebook), considerando que la misma es utilizada por usuarios AM y desde tablets. Mantener en mente este objetivo es posible debido a que las RD conducen todo el CP. 


\subsubsection{Ejecución paso a paso del CP centrado en AM}

\section{Entrada (recomendaciones de diseño)}

Ya se señaló que el CP es dirigido por nuestras RD desde el punto de entrada inicial y hasta el punto de salida, que entrega un prototipo final.

\section{Paso 1 (1.1. Declarar suposiciones/1.2. Definir kit de prueba/1.3. Declarar hipótesis)}

En la actividad 1.1, se elabora una lista de suposiciones enfocada en el usuario AM.

La lista de suposiciones se declara a partir de la hoja de suposiciones de usuario AM, considerando las $\mathrm{RD}$ que reflejan las necesidades y las preferencias de los usuarios AM. Por ejemplo, la figura I ilustra la pregunta 5 de la hoja de suposiciones de usuario AM resultante.

Figura I. Hoja de suposiciones de usuario AM

\section{Hoja de suposiciones de usuario AM}

5. Respecto a los iconos usados para acceder a las distintas funcionalidades: ¿el tamaño de los mismos es acorde a las necesidades de los usuario?, ¿son los mismos (o similares) a los de otras redes sociales/aplicaciones?, ¿evidencian la funcionalidad para la que se los usa? y ¿están situados en una barra de pestañas visible y de fácil acceso en todo momento?

Fuente: elaboración propia

Así, por ejemplo, en el cuadro I, se presenta la suposición 5 de la lista de suposiciones declarada a partir de la hoja de suposiciones de usuario AM.

Cuadro I. Suposición 5 de la lista de suposiciones

Número de

pregunta

\section{Suposición}

5. Los AM tienen dificultad para cliquear en los iconos debido a que el tamaño de los mismos es, en general, pequeño para asociar un icono con su correspondiente funcionalidad, es decir, no todos los iconos evidencian su funcionalidad y presentan dificultad para localizar las opciones de búsqueda y/o filtro.

Fuente: elaboración propia. 
(cont.) Paso 1.

En la actividad 1.2, se define un kit de prueba, el cual será ejecutado por los usuarios AM para evaluar los prototipos de baja y de alta fidelidad en los bucles 1 y 2 del CP, respectivamente. El kit de prueba propuesto se compone de las siguientes seis tareas:

1. Iniciar sesión/cerrar sesión.

2. Buscar un contacto.

3. Enviar mensaje

4. Realizar publicación.

5. Ver notificación.

6. Aceptar solicitud de amistad.

El cuadro II presenta la tarea 4 del kit de prueba. Cada tarea se compone de un conjunto de acciones que han de ejecutar los usuarios AM.

Las columnas tres y cuatro permiten asociar el prototipo (de baja o de alta fidelidad) con la acción que hay que ejecutar. Esta asociación divide el kit de prueba en dos conjuntos de tareas y acciones que han de ejecutar los usuarios AM para cada uno de los bucles del CP.

El cuadro III muestra la planilla de evaluación, que se utiliza para registrar los resultados de ejecutar las tareas (y sus acciones) por parte de los usuarios AM, utilizando Facebook y los prototipos de baja y alta fidelidad desde tablets.

Cuadro II. Tarea 4 del kit de prueba

\begin{tabular}{|c|c|c|c|c|c|}
\hline Tarea & $\begin{array}{c}\text { Acción que hay } \\
\text { que evaluar }\end{array}$ & $\begin{array}{c}\text { Prototipo } \\
\text { de baja } \\
\text { fidelidad }\end{array}$ & $\begin{array}{c}\text { Prototipo } \\
\text { de alta } \\
\text { fidelidad }\end{array}$ & $\begin{array}{l}\text { Suposición } \\
\text { asociada }\end{array}$ & $\begin{array}{c}\text { Recomendación } \\
\text { aplicada }\end{array}$ \\
\hline $\begin{array}{l}\text { 4. Realizar } \\
\text { publicación }\end{array}$ & $\begin{array}{l}\text { Identificar y seleccionar el } \\
\text { botón para definir el tipo } \\
\text { de publicación (escribir } \\
\text { texto o grabar audio). } \\
\text { Escribir la publicación. } \\
\text { Grabar la publicación. } \\
\text { Identificar y seleccionar el } \\
\text { botón para publicar. } \\
\text { Identificar y seleccionar el } \\
\text { botón para eliminar. } \\
\text { Identificar y seleccionar el } \\
\text { icono para retornar a la pá- } \\
\text { gina principal. }\end{array}$ & $x$ & $\begin{array}{l}x \\
x\end{array}$ & $\begin{array}{c}1,2,3 \\
4 \text { y } 5\end{array}$ & $\begin{array}{l}1.1,1.2,2.1 \\
2.2,2.3,3.1 \\
3.3,3.5,3.7 \\
3.8,4.1,4.2, \\
4.3,4.4,4.5 \\
5.1,5.2,5.3 \\
5.4 \text { y } 5.5\end{array}$ \\
\hline
\end{tabular}

Fuente: elaboración propia. 
(cont.) Paso 1.

\section{Cuadro III. Planilla de evaluación}

\begin{tabular}{l|l}
\multicolumn{1}{c|}{ Criterios } & \multicolumn{1}{c}{ Valores } \\
\hline Sexo & Femenino/Masculino. \\
\hline Edad & Numérico. \\
\hline Tarea & Indicar la tarea evaluada. \\
\hline Grado de dificultad & Fácilmente/Con Asistencia/No la realizó. \\
\hline Tipo de dificultad & $\begin{array}{l}\text { Se desorientó/No lo encontró/No lo entendió/Problemas con objetivos táctiles } \\
\text { (identificación-selección). }\end{array}$ \\
\hline Grado de satisfacción & Alto/Medio/Bajo (estos valores están asociados al grado de dificultad). \\
\hline Comentarios & Texto. \\
\hline
\end{tabular}

Fuente: elaboración propia.

En la actividad 1.3, por cada suposición de la lista de suposiciones se declara una hipótesis de la lista de hipótesis. Por ejemplo, a partir de la suposición 5, en el cuadro IV se declara la hipótesis 5 y se define su respectiva validación a partir del kit de prueba.

Cuadro IV. Hipótesis 5 de la lista de hipótesis

\begin{tabular}{l|l} 
Número de & \multicolumn{1}{c}{ Hipótesis } \\
suposición & \\
\hline 5 & Un diseño de IU de una red social que incluye iconos: \\
- Similares a los utilizados por otras redes sociales/aplicaciones. \\
- De tamaño mediano/grande. \\
- De imagen estática, acompañada con una descripción textual de su funcionalidad. \\
- Situados en una barra de menú de navegación visible con tamaño amplio y de fácil \\
acceso. \\
Incrementará la frecuencia de uso por parte de los usuarios AM. \\
Validación: la hipótesis 5 se verifica cuando los usuarios AM identifiquen y seleccionen \\
adecuadamente los iconos requeridos para ejecutar todas las tareas del kit de prueba.
\end{tabular}

Fuente: elaboración propia.

Es necesario describir al grupo de usuarios AM en los que se centra la ejecución del CP. Para ello usamos el método de protopersonas que aplica Lean UX (Gothelf y Seiden, 2014). 
(cont.) Paso 1.

\section{Usuarios AM como personas}

Los usuarios en los que se centra el desarrollo son 32 AM residentes en la región patagónica que asisten al taller (Saldaño et al., 2018a) desarrollado en el marco del Proyecto de Extensión y Vinculación. El rango de edades oscila entre los 65 y los 85 años y predomina el sexo femenino (29 mujeres y 3 hombres). Como característica común, todos manifiestan que han tomado contacto con el mundo de la tecnología con ayuda de sus hijos o nietos. A partir de una intensa interacción con los 32 AM participantes, se definen tres modelos de personas: Carmen, Claudio y María. La figura II, por ejemplo, nos informa sobre Carmen.

Figura II. Modelo de personas: usuario AM Carmen

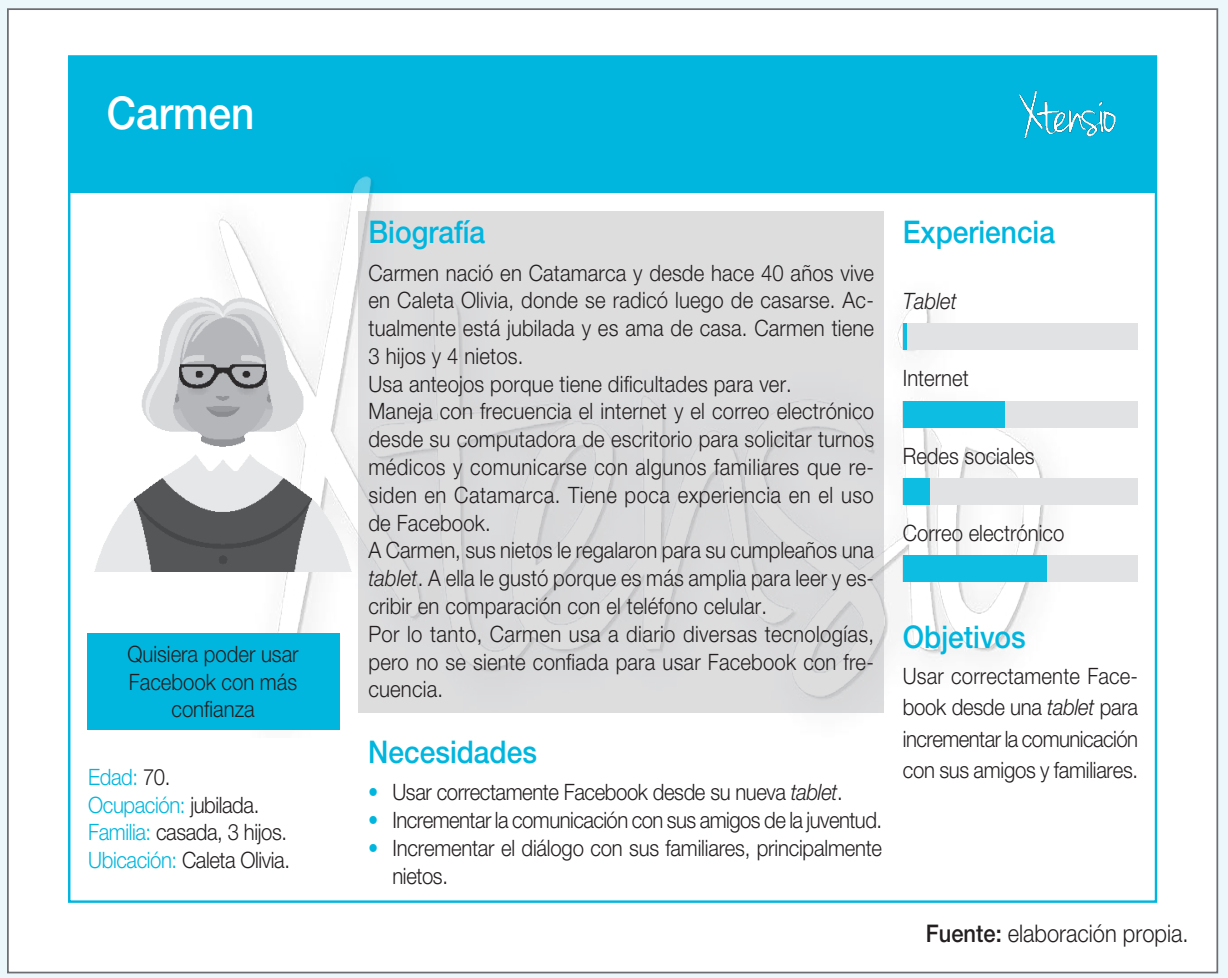

\section{Tareas y resultados esperados}

Lean UX propone definir funciones y, por cada una de estas funciones, los resultados que se esperan alcanzar para satisfacer a los usuarios.

En nuestra propuesta, las tareas del kit de prueba son las encargadas de alcanzar este objetivo. El cuadro V presenta los resultados esperados para la tarea 4 del kit de prueba. 
(cont.) Paso 1.

\section{Cuadro V. Resultados esperados de la tarea 4}

\begin{tabular}{|c|c|}
\hline Tarea & Resultados esperados \\
\hline $\begin{array}{l}\text { 4. Realizar } \\
\text { publicación }\end{array}$ & $\begin{array}{l}\text { Incrementar el número de usuarios AM que puedan realizar con éxito las siguientes } \\
\text { acciones: } \\
\text { - Acceder a la interfaz que permite realizar la publicación. } \\
\text { - Ingresar los datos (escribir texto/grabar audio) de la publicación. } \\
\text { - Identificar y seleccionar cada uno de los iconos/botones requeridos para la eje- } \\
\text { cución de la tarea. } \\
\text { - Completar la tarea con éxito. } \\
\text { - Retornar a la página principal. }\end{array}$ \\
\hline
\end{tabular}

Fuente: elaboración propia.

Ejecutadas las actividades del paso 1, se inicia el bucle 1 del CP.

\section{Bucle 1 (pasos 1, 2 y 3 del CP)}

Concretado el paso 1, en el paso 2 se propone un primer prototipo de baja fidelidad. El diseño se enfoca en la presentación que hay que trasmitir a los usuarios AM, definiendo la distribución, la apariencia visual y el tamaño de los elementos de las IU requeridas por cada una de las tareas. En el paso 3, este prototipo es evaluado por los usuarios AM, a quienes se les dirige y observa al ejecutar las acciones indicadas por el kit de prueba para cada una de las tareas. Los resultados se registran utilizando la planilla de evaluación (véase cuadro III), cuyo feedback se utiliza para validar el listado de hipótesis y determinar la finalización del bucle 1 con la entrega del prototipo de baja fidelidad final. La figura III presenta el diseño de la IU de baja fidelidad final para la tarea 4. Particularmente, en la figura III se resaltan las $\mathrm{RD}$ de la categoría 5, que se aplican en la definición de la IU para la tarea 4. Es importante observar, por ejemplo, que para definir el icono de Inicio necesario para la acción «ldentificar y seleccionar el icono para retornar a la página aprincipal» se han aplicado las RD 5.1, 5.3, 5.4 y 5.5 de la categoría 5 del cuadro I.

Figura III. Prototipo de IU de baja fidelidad para la tarea 4

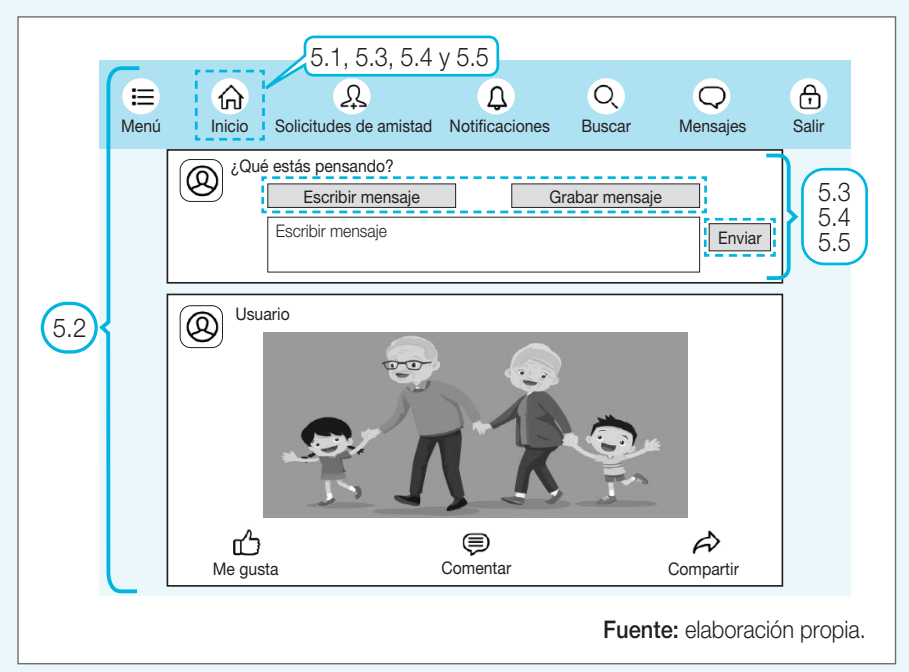




\section{Bucle 2 (pasos 1, 4 y 5 del CP)}

En el paso 4, incorporando el diseño de la presentación de las IU del prototipo de baja fidelidad final, se propone un prototipo de alta fidelidad cuyo diseño se enfoca en incorporar las funcionalidades básicas requerida por cada una de las tareas. En el paso 5, este prototipo es evaluado por los usuarios AM y, nuevamente, de la misma manera que en el bucle 1, hasta que con el feedback obtenido se determine la finalización del bucle 2. La figura IV presenta el diseño de la IU de alta fidelidad final para la tarea 4, que se entrega a la «salida» del CP. Particularmente, en la figura IV, se resaltan las RD de la categoría 5, que se aplican en la definición de la IU para la tarea 4. Es importante observar, por ejemplo, que para definir los iconos de la barra de pestañas se siguen manteniendo en mente las RD 5.1, 5.3, 5.4 y 5.5 de la categoría 5 del cuadro 1.

También, se observa que los iconos de Notificaciones y Salir, situados en la barra de herramientas, han sido reemplazados para reforzar el diseño skeumorphism y affordance (según prescribe la categoría 5 de nuestras RD). Esta mejora, relacionada con la apariencia visual de elementos de la IU, se realiza haciendo uso de las ventajas que ofrece una herramienta para desarrollar prototipos de alta fidelidad. Además, en la figura IV se observa la inclusión del botón Eliminar en el prototipo de alta fidelidad. Este botón se incluye para dar respuesta a reiterados comentarios de los usuarios AM (registrados en la «planilla de evaluación» del cuadro III) acerca de la necesidad de mantener visible y con fácil acceso la opción de eliminar una publicación.

Figura IV. Prototipo de IU de alta fidelidad para la tarea 4

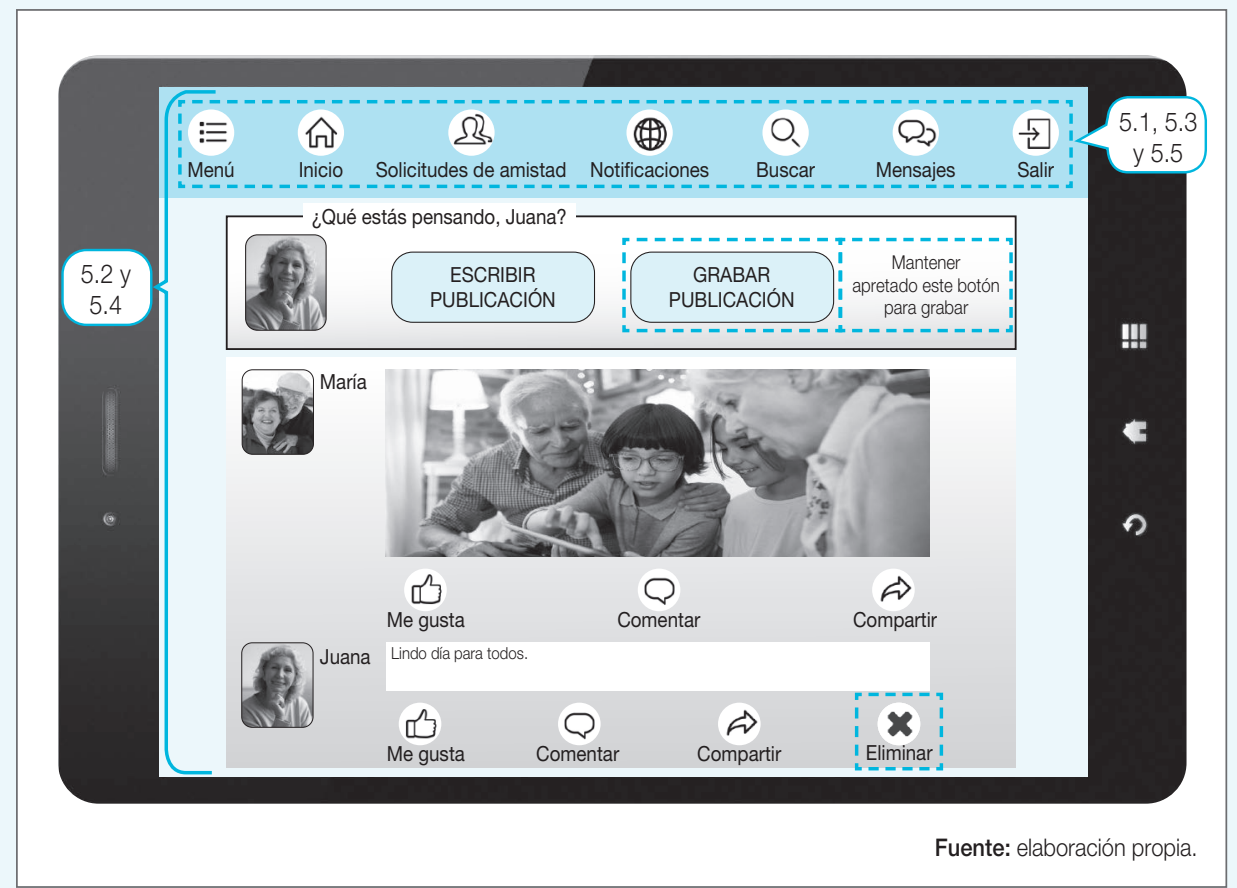


Tal como ya señalamos, las cinco categorías de las RD dirigen el CP y las decisiones de diseño en el desarrollo de ambos prototipos. Sin embargo, y debido a que, por razones de espacio, en este estudio solo se ha trabajado en ambos prototipos con la categoría 5 (áreas y elementos objetivo de las RD), el efecto de aplicar las prescripciones de esta categoría en particular se hace más evidente en el prototipo de baja fidelidad (que se focaliza en la presentación que hay que trasmitir a los usuarios AM) que en el prototipo de alta fidelidad (que se focaliza en las funcionalidades básicas que hay que ofrecer a los usuarios AM).

\section{Resultados obtenidos y discusión}

Como se señalaba con anterioridad, la evaluación de Facebook y de los prototipos de baja y alta fidelidad por parte de los usuarios AM se lleva a cabo aplicando el kit de prueba y registrando los resultados en la planilla de evaluación. Los 32 usuarios AM participantes tienen un rango de edad que oscila entre los 69 y los 86 años y predomina el sexo femenino (18 mujeres y 2 hombres). Los resultados finales se reflejan en tres cuadros, uno por cada producto evaluado: Facebook, prototipo de baja fidelidad final y prototipo de alta fidelidad final. A continuación, el cuadro 3 combina y presenta solo los resultados obtenidos de la evaluación de la tarea 4 para los tres productos evaluados.

\section{Cuadro 3. Resultados de la ejecución de la tarea 4}

\begin{tabular}{|c|c|c|c|c|c|c|c|c|}
\hline \multirow[b]{3}{*}{ Tarea } & \multicolumn{3}{|c|}{ Grado de dificultad } & \multicolumn{5}{|c|}{ Tipo de dificultad } \\
\hline & \multirow{2}{*}{ Fácilmente } & \multirow{2}{*}{$\begin{array}{c}\text { Con } \\
\text { asistencia }\end{array}$} & \multirow{2}{*}{$\begin{array}{l}\text { No la } \\
\text { realizó }\end{array}$} & \multirow{2}{*}{$\begin{array}{c}\text { Se } \\
\text { desorientó }\end{array}$} & \multirow{2}{*}{$\begin{array}{c}\text { No lo } \\
\text { encontró }\end{array}$} & \multirow{2}{*}{$\begin{array}{l}\text { No lo } \\
\text { entendió }\end{array}$} & \multicolumn{2}{|c|}{$\begin{array}{l}\text { Problemas con } \\
\text { objetivos táctiles }\end{array}$} \\
\hline & & & & & & & Identif. & Selec. \\
\hline Facebook & 6 & 22 & 4 & 3 & 7 & 3 & 7 & 6 \\
\hline $\begin{array}{l}\text { Prototipo de } \\
\text { baja fidelidad }\end{array}$ & 22 & 10 & 0 & 6 & 4 & 0 & 0 & 0 \\
\hline $\begin{array}{l}\text { Prototipo de } \\
\text { alta fidelidad }\end{array}$ & 30 & 2 & 0 & 2 & 0 & 0 & 0 & 0 \\
\hline
\end{tabular}

Fuente: elaboración propia.

El cuadro 3 pone en evidencia que los prototipos desarrollados por nuestro CP han logrado alcanzar los resultados esperados de la tarea 4 que se presentaron en el cuadro $\mathrm{V}$ al 
incrementar notablemente el número de usuarios AM que pudieron realizar con éxito las acciones correspondientes a la tarea 4. Por ejemplo, observando los resultados de la columna "Grado de dificultad», en Facebook, el $12 \%$ de los usuarios AM realizó la tarea 4 «fácilmente», el $69 \%$ la realizó «con asistencia» y el 12\% «no la realizó», mientras que, en el prototipo de baja fidelidad final, el $69 \%$ de los usuarios AM realizó la tarea 4 «ácilmente», el $31 \%$ la realizó «con asistencia» y ninguno de los 32 usuarios $A M$ «no la realizó». Y, por último, en el prototipo de alta fidelidad final, el $94 \%$ de los usuarios AM realizó la tarea 4 «fácilmente» y el $6 \%$ la realizó «con asistencia». En el cuadro 3, otro aspecto relevante que hay que señalar es la reducción al $0 \%$ de los usuarios AM que tuvieron «problemas con objetivos táctiles» en los prototipos de baja y alta fidelidad finales. Esto es debido a la aplicación de las $\mathrm{RD}$ de la categoría 5 (áreas y elementos objetivo) que presenta el cuadro 1, que dieron la solución a los problemas de identificación y selección de los objetivos táctiles por parte de los usuarios AM.

Cabe destacar que, a partir de las tres tablas obtenidas al evaluar Facebook y de los prototipos de baja y de alta fidelidad finales, los resultados fueron analizados de diferentes maneras para determinar si el diseño alternativo de red social (desarrollado por nuestro CP para tablets) mejora la experiencia de los usuarios AM. Por ejemplo, la figura 5 asocia el "grado de dificultad» (fácilmente/con asistencia/no la realizó) experimentado por los usuarios $\mathrm{AM}$ al ejecutar la tarea 4 con el "grado de satisfacción» (alto/medio/bajo) para los tres productos evaluados. Creemos que esta asociación es posible porque, si un usuario AM no experimenta dificultades al ejecutar las acciones que componen una tarea, se fortalece su confianza y seguridad en el uso del producto. De esta manera, el usuario AM va a estar más satisfecho si alcanza con éxito el objetivo buscado, el cual, para el caso que hemos utilizado como ejemplo, consiste en poder realizar una publicación.

Figura 5. «Grado de satisfacción» asociado al "grado de dificultad»

Tarea 4. Realizar publicación

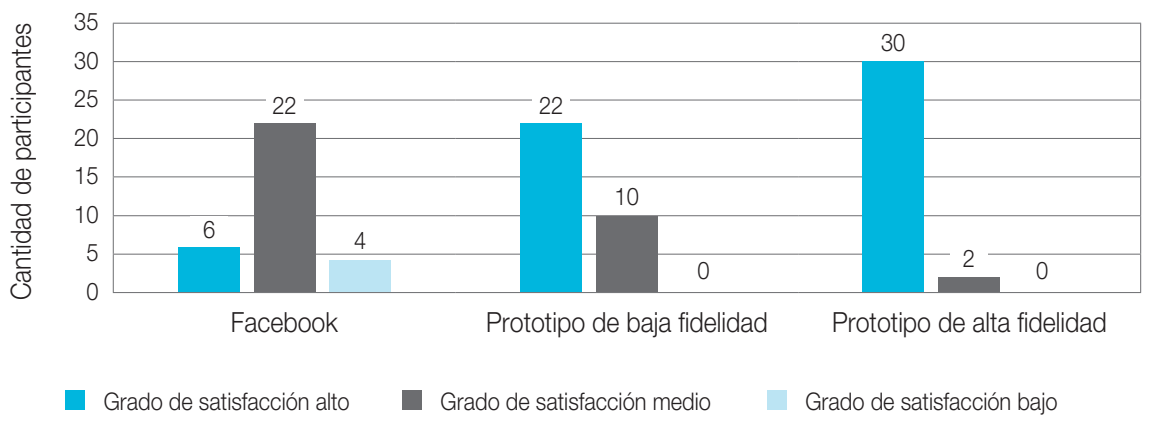

Fuente: elaboración propia 
De esta manera, los resultados obtenidos constituyen un avance en el uso de las redes sociales por parte de los AM a la luz de los trabajos relacionados, estudiados y analizados.

Avanzando en la línea de investigación propuesta en Miranda et al. (2014) en el presente trabajo, los usuarios AM logran interactuar con un prototipo de alta fidelidad. Respecto al proyecto Enred@te presentado por la TECSOS destacamos que el perfil de los usuarios AM que participaron en la realización del kit de prueba no está limitado a una organización/ fundación específica, sino que está enfocado en los AM interesados en el uso de las redes sociales. Analizada la plataforma +Simple, destacamos los logros alcanzados por los AM del actual trabajo al tener la posibilidad de interactuar con un diseño alternativo de red social para Facebook, enfocado en mejorar la UX de los AM desde tablets.

En Sulmont et al. (2018) se identifican importantes barreras en el uso de Facebook pero con una cantidad limitada de AM. Es así que para el actual trabajo se realizó un análisis exhaustivo con la finalidad de determinar el número de AM participantes, resultando 32 AM.

En Gomes et al. (2014) los resultados muestran que las tareas son más fáciles de realizar en el prototipo que en la aplicación nativa de Facebook. De manera similar, logramos demostrar que el diseño de un prototipo de interfaz adaptado a las necesidades de los AM permite mejorar la interacción de los AM con la red social Facebook desde tablets. Por ejemplo, se puede establecer un paralelo entre la tarea «Post» de Gomes et al. (2014) y las tareas «Enviar mensaje» y «Realizar publicación» de nuestro trabajo. En ambos casos se evidencia una mejora en la UX al realizar la tarea en el prototipo final respecto de Facebook, permitiendo que las mismas sean realizadas por casi la totalidad de los AM participantes en ambos trabajos.

\section{Conclusiones y trabajos futuros}

Es un hecho que las personas prefieren los dispositivos móviles a la hora de interactuar en su vida cotidiana. Particularmente, las redes sociales constituyen un instrumento digital vital para la integración ciudadana, social y también familiar, asistiendo a la superación de brechas generacionales y geográficas.

En este contexto, los usuarios AM no son ajenos a esta tendencia mundial y, en particular, cuando se trata de acceder a una red social desde un dispositivo móvil, prefieren hacerlo desde uno de tipo tablet. Estas herramientas digitales son vitales para la integración de los usuarios AM. No obstante, muchos usuarios AM manifiestan frustración y falta de uso frecuente de las mismas, debido a que los diseños IU no están pensados para brindar la confianza y seguridad que ellos necesitan. 
En este trabajo, tal como se mencionó en el apartado 3.1, se propone un conjunto de 23 RD para mejorar la experiencia de los usuarios AM en redes sociales y desde tablets. Cada una de las cinco categorías propuestas en las RD se enfoca en mejorar la UX desde diferentes aspectos, tales como ofrecer una interfaz más simple, brindar un entorno intuitivo, mitigar dificultades de visión y motoras, facilitar la navegación en la página, mejorar la disposición de los elementos objetivo en la pantalla del dispositivo y la aplicación de diseño skeumorphism y affordance.

Posteriormente, se define y aplica un CP iterativo e incremental, dirigido por nuestras RD y basado en la metodología Lean UX (Gothelf y Seiden, 2014), centrado en los AM (véase apartado 3.2).

Las pruebas efectuadas ponen en evidencia que el diseño alternativo de red social propuesto para tablets permite a los usuarios AM:

1. Reducir notablemente el grado y el tipo de dificultades que experimentan al usar la red social Facebook.

2. Incrementar el número de tareas (y sus respectivas acciones) ejecutadas con éxito.

3. Debido a los puntos 1 y 2 se logra incrementar el grado de satisfacción y, por ende, contribuir a mejorar la UX.

Estos resultados pueden observarse en el cuadro 3 del apartado 4 de este trabajo.

Sin embargo, aún existen algunos aspectos que requieren proseguir con este trabajo, tales como:

- Enriquecer los diseños de los prototipos para incluir otras tareas que no fueron contempladas en esta investigación.

- Extender las pruebas, incluyendo otros grupos de usuarios AM.

- Seguidamente, implementar el prototipo de alta fidelidad final como una alternativa concreta de red social para que sea utilizado por los usuarios AM desde tablets.

Asimismo, cabe señalar que nuestra propuesta, compuesta por RD + CP, sienta las bases para customizar, extender y/o generalizar la aplicación de la misma al diseño y al desarrollo de otros productos destinados a usuarios pertenecientes a la población AM. 


\section{Referencias bibliográficas}

Amaya Balaguera, Y. D. (2013). Metodologías ágiles en el desarrollo de aplicaciones para dispositivos móviles. Estado actual. Revista de Tecnología-Journal of Technology, 12(2), 111-124. Recuperado de <https: //dialnet.unirioja.es/servlet/articulo?codi go $=6041502>$ (consultado el 11 de diciembre de 2019).

Barroso Osuna, J. y Aguilar Gavira, S. (2017). Uso e interés por las redes sociales entre las personas mayores desde una perspectiva de género. Un estudio en las universidades de mayores andaluzas. Revista Tecnología, Ciencia y Educación, 7, 51-71. Recuperado de <https://www.tecnologiaciencia-educacion.com/index.php/TCE/ issue/view/18> (consultado el 11 de diciembre de 2019).

Blank, S. (2013). The Four Steps to the Epiphany. K\&S Ranch.

Brown, T. (2008). Design Thinking. Harvard Business School Publishing Corporation.

Budiu, R. y Nielsen, J. (2015). User Experience for Mobile Applications and Websites. Design Guidelines for Improving the Usability of Mobile Sites and Apps (3. ${ }^{a}$ ed.). Fremont, California, EE. UU.: Nielsen Norman Group.

Cardozo, C., Martín, A. y Saldaño, V. (2017a). Abuelos del siglo XXl: una propuesta para mejorar la experiencia en el uso de redes sociales desde dispositivos móviles. CONAIISI 2017, 2 y 3 de noviembre de 2017 (pp. 478487). Universidad Tecnológica Nacional/Facultad Regional Santa Fe, Argentina.

Cardozo, C., Martín, A. y Saldaño, V. (2017b). Los adultos mayores y las redes sociales: analizando propuestas para mejorar la interacción. Informe Científico Técnico UNPA (ICT-UNPA), 9(2), 1-29.
Cornejo, R., Weibel, N., Tentori, M. y Favela, J. (2015). Promoting active aging with a paperbased SNS application. Proceedings 9th International Conference on Pervasive Computing Technologies for Healthcare (Pervasive Health '15) (pp. 209-212). Estambul, Turquía: Institute for Computer Sciences, Social-Informatics and Telecommunications Engineering (ICST).

Duggan, M. (19 de agosto de 2015). The demographics of social media users. Pew Research Center: Internet \& Technology. Recuperado de <http://www.pewinternet. org/2015/08/19/the-demographics-ofsocial-media-users/> (consultado el 21 de septiembre de 2019).

Flamarich Zampalo, J. (2013). Diseño de interfaces para dispositivos móviles. Barcelona: Fundación para la Universitat Oberta de Catalunya.

Gomes, G., Duarte, C., Coelho, J. y Matos, E. (2014). Designing a Facebook interface for senior users. The Scientific World Journal, 2014, 1-8.

Gothelf, J. y Seiden, J. (2014). Lean UX: cómo aplicar los principios Lean a la mejora de la experiencia de usuario. Logroño, España: Universidad Internacional de La Rioja, SA.

INDEC. (2019). Recuperado de <https://www. indec.gob.ar> (consultado el 22 de septiembre de 2019).

Instituto Nacional de Servicios Sociales para Jubilados y Pensionados. (2017). Programa UPAMI. Recuperado de <https://www.pami. org.ar/servicios/actividades-preventivas> (consultado el 2 de septiembre de 2019).

Leme, R., Zaina, L. y Casadei, V. (2015). A study of elderly users' interaction with Facebook on mobile devices: identification 
and definition of the personas. Proceedings of the 14th Brazilian Symposium on Human Factors in Computing Systems (pp. 1-10). Salvador, Brasil: ACM New York. Recuperado de <https://www.think mind. org/index. php? view=instance\&inst ance $=\mathrm{ACH}+2014>$ (consultado el 11 de diciembre de 2019).

Lu, K. (12 de junio de 2017). Growth in mobile news use driven by older adults. Pew Research Center. Recuperado de <http://www. pewresearch.org/fact-tank/2017/06/12/ growth-in-mobile-news-use-driven-byolder-adults/> (consultado el 5 de febrero de 2019).

Luna García, H., Mendoza González, R. y Álvarez Rodríguez, F. (2015). Patrones de diseño para mejorar la accesibilidad y uso de aplicaciones sociales para adultos mayores. Comunicar, XXII(45), 85-94. doi: https://doi. org/10.3916/C45-2015-09.

Marcelino, I., Laza, R. y Pereira, A. (2016). SSN: senior social network for improving quality of life. International Journal of Distributed Sensor Networks, 12(7), 1-8. doi: https:// doi.org/10.1177/155014772150734.

Miranda, M. G., Martín, A., Saldaño, V. y Gaetán, G. (2014). Usabilidad y accesibilidad en las redes sociales: una experiencia de usuarios adultos mayores en Facebook. Informe Científico Técnico UNPA (ICT-UNPA), 6(2), 118-140.

Nielsen, J. (2013). Tablet Website and Application UX (3. ${ }^{\mathrm{a} e d}$.). Fremont, California, EE. UU.: Nielsen Norman Group.

Peñalver Blanco, A. (2016). Buenas prácticas del proyecto piloto Enred@te: red social digital para personas mayores y voluntariado de la Cruz Roja Española. Revistas Científicas Complutenses, 29(2), 201-212. Recuperado de <http://revistas.ucm.es/in dex.php/CUTS/article/view/51656> (consultado el 11 de diciembre de 2019).
Pernice, K., Estes, J. y Nielsen, J. (2013). Senior Citizens (Ages 65 and Older) on the Web (2. ${ }^{a}$ ed.). Fremont, California, EE. UU.: NN/g Nielsen Norman Group.

Presidencia de la Nación. (2016). Secretaría de Modernización: +Simple. Recuperado de $<$ https://www.massimple.gob.ar/> (consultado el 5 de febrero de 2019).

Ries, E. (2011). The Lean Startup: How Today's Entrepreneurs Use Continuous Innovation to Create Radically Successful Businesses. Nueva York, EE. UU.: Crown Business.

Saldaño, V., Gaetán, G., Martín, A. y Cardozo, C. (2018a). Abuelos del siglo XXI: taller de redes sociales para adultos mayores. Caleta Olivia, Santa Cruz, Argentina: UNPA-PEyV 2 (perteneciente al PgEyV: Observatorio de Experiencia de usuario, UNPA, expediente núm. 29.820/18).

Saldaño, V., Gaetán, G., Martín, A. y Cardozo, C. (2018b). Tallerparaadultosmayores: ¿cómo usar los nuevos teléfonos móviles? Caleta Olivia: UNPA-PEyV 1 (pertenecienteal PgEyV: Observatorio de experiencia de usuario, UNPA, expediente núm. 29.820/18).

Saldaño, V., Martín, A., Gaetán, G. y Vilte, D. (2013). Web accessibility for older users: a southern argentinean view. The Eighth International Conference on Software Engineering Advances (ICSEA) (pp. 621-626). Italia: ICSEA 2013 Editors.

Sulmont, E., Pontual Falcão, T. y Moffatt, K. (2 de febrero de 2018). Designing and evaluating an alternative Facebook interface for older adults. Montreal, Canadá: School of Information Studies, McGill University. doi: https://doi.org/10.1155/2014/741567.

UNPA. (2010). Grupo de Investigación: GIFIS. Instituto de TecnologíaAplicada(ITA). Recuperado de <https://www.unpa.edu.ar/cecyt/ 1876/grupo/gifis> (consultado el 11 de diciembre de 2019). 
Vilte, D., Saldaño, V., Gaetán, G. y Martín, A. (2015). Identificando barreras en la interacción conFacebook: una experiencia conadultos mayores de la Patagonia Austral. Informe Científico Técnico UNPA (ICT-UNPA), 7(2), 249-268.

Vivanco, M. (2005). Muestreo estadístico. Diseño y aplicaciones. Santiago de Chile, Chile: Editorial Universitaria.
Warfel, T. (2009). Prototyping: A Practioner's Guide. Brooklyn, Nueva York, EE. UU: Rosenfeld Media.

World Wide Web Consortium (W3C). (22 de junio de 2018). Web content accessibility guidelines (WCAG). W3C-Web Accessibility Initiative (WAl). Recuperado de <https:// www.w3.org/WAl/intro/wcag> (consultado el 21 de septiembre de 2019).

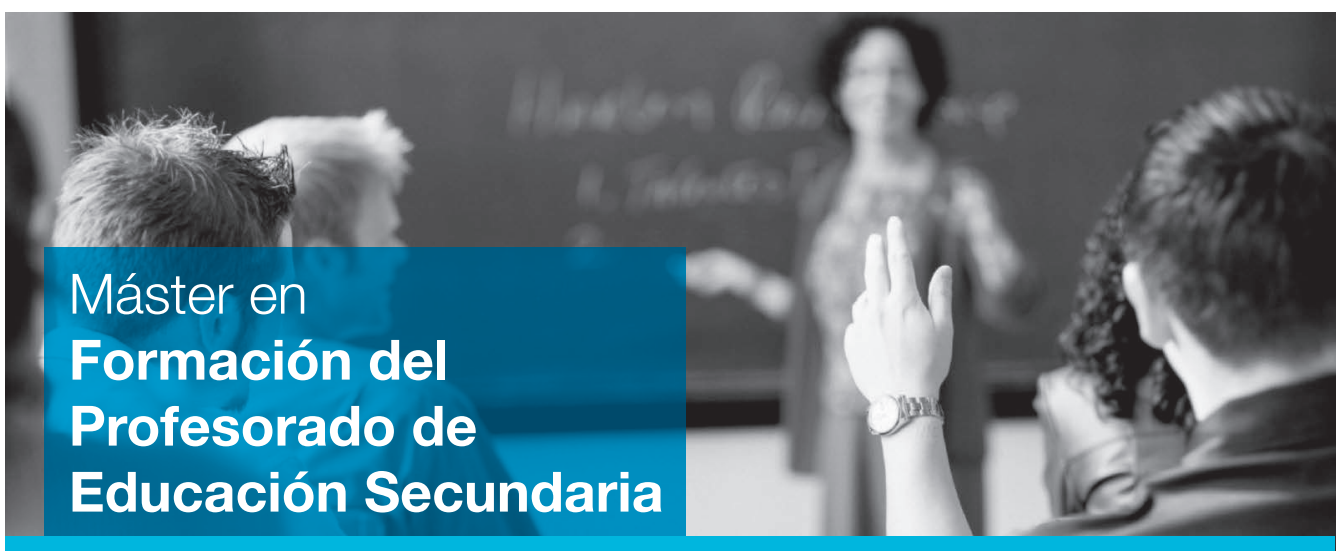

Este máster oficial en Formación del Profesorado de Educación Secundaria Obligatoria, Bachillerato, Formación Profesional y Enseñanza de Idiomas [60 créditos ECTS] tiene una duración normal de 12 meses.

Dirigido a: La universalización de la enseñanza secundaria y el incremento de la atención a la diversidad de alumnos en todos los niveles de enseñanza han hecho más patente la necesidad de mayor formación didáctica. El educador ya no solo ha de ser un experto en su materia, sino que debe tener la suficiente capacidad didáctica para adaptar la misma a grupos de alumnos muy heterogéneos en intereses, capacidades y actitudes.

Objetivos: Adquirir todas las habilidades y competencias necesarias para poder desarrollar una carrera profesional en el ámbito de la enseñanza en los niveles de Educación Secundaria Obligatoria y Bachillerato, Formación Profesional y Enseñanzas de Idiomas de acuerdo a la normativa vigente, ya sea para dar clase en centros públicos, privados o concertados. Ofrecer formación integral y especializada a los participantes. 